\title{
Recommendations on quantities, symbols and measurement units for publications in ACQUAL: reporting measurement uncertainty in chemical analysis
}

\author{
Wolfgang Richter
}

Received: 5 December 2007 / Accepted: 7 December 2007/Published online: 30 January 2008

(C) Springer-Verlag 2008

Measurement uncertainty is an integral part of a measurement result. Without a statement of uncertainty a measurement result is not complete. Concluding about compatibility with other measurement results obtained for the same measurand or with compliance limits is not possible and the measurement result does therefore, not serve its purpose. The measurement uncertainty is a quality criterion, which is important for the use of the result, in particular if decisions are to be based on that result. The Guide to the Expression of Uncertainty in Measurement (GUM), ISO 1995, and the CITAC/EURACHEM document Quantifying Uncertainty in Analytical Measurement (QUAM), 2001, an introduction to the GUM for chemists, are useful guides for evaluating measurement uncertainty in chemical analysis. These guides and their recommendations on how to report measurement results together with the associated uncertainty serve as the basis for the following.

The final measurement result obtained after corrections for all known systematic effects is regarded as the best estimate $y$ of the measurand $Y$ within the range of values which could reasonably be attributed to the measurand under the given conditions of measurement. It is reported in the form:

$Y=y \pm U \quad$ with $\quad U=k u(y)$

or

$Y=y\left(1 \pm U_{\mathrm{r}}\right) \quad$ with $\quad U_{\mathrm{r}}=U / y$.
$U$ is the so-called expanded uncertainty, $U_{\mathrm{r}}$ the relative expanded uncertainty, $u(y)$ the combined standard uncertainty associated with the final measurement result obtained by the evaluation procedures described in the above mentioned (and other) guides and $k$ the coverage factor. $U$ is the half width of an interval about the best estimate $y$ that encompasses a large fraction of the probability distribution characterized by $y$ and $u(y)$ at a desired level of confidence (or coverage probability). $k=2$ is often chosen in chemical measurement which corresponds approximately to a coverage probability of 0.95 in many cases of analytical practice. (The distinction in notation between measurands and their best estimates, here $Y$ and $y$, respectively, is, according to the GUM, only used in general expressions but not with actual quantity symbols).

If the number of effective degrees of freedom is known a better approximation is using for $k$ the tabulated value of the $t$-distribution corresponding to this number of degrees of freedom and the desired coverage probability.

The number of digits of the numerical value of the result should be consistent with the uncertainty. $U$ and $u(y)$ are sufficiently described with two significant digits.

In the box below two examples are given of how chemical measurement results can be reported together with the associated measurement uncertainty.

W. Richter $(\bowtie)$

Braunschweig, Germany

e-mail: wolfgang.k.richter@t-online.de 


\section{Examples of reporting chemical measurement results with associated uncertainty:}

(a) The mass concentration of lead in the waste water sample is $\gamma(\mathrm{Pb})=(12.04 \pm 0.43) \mathrm{mg} / \mathrm{L}, k=2$, coverage probability approximately 0.95 .

If the relative uncertainty is to be given, the following form is recommended:

$\gamma(\mathrm{Pb})=12.04 \mathrm{mg} / \mathrm{L}$, relative expanded uncertainty $U_{\mathrm{r}}=0.036(=3.6 \%), k=2$, coverage probability approximately 0.95

which is equivalent to

$\gamma(\mathrm{Pb})=12.04(1 \pm 0.036) \mathrm{mg} / \mathrm{L}, k=2$, coverage probability approximately 0.95 .

(b) The mass fraction of sulphur in the coal sample is

$w(\mathrm{~S})=(7.83 \pm 0.32) \mathrm{mg} / \mathrm{g}, \quad k=2.23$, coverage probability $0.95, t$-distribution for 10 degrees of freedom. The relative expanded uncertainty is $0.041(=4.1 \%)$.

Alternative, like under (a):

$w(\mathrm{~S})=7.83(1 \pm 0.041) \mathrm{mg} / \mathrm{g}, k=2.23$, coverage probability $0.95, t$-distribution for 10 degrees of freedom. 